\title{
Advocacy, Communication, Chain of Command, and Evidence-Based Practice
}

A positive childbirth experience fulfills or exceeds a woman's prior personal and sociocultural beliefs and expectations, including giving birth to a bealthy baby in a clinically and psychologically safe environment with continuity of practical and emotional support from a birth companion(s) and kind, technically competent clinical staff.

\section{INTRODUCTION}

A safe childbirth is a physiologic process that is accomplished without complications (World Health Organization [WHO], 2018). Safety requires nursing advocacy, communication, appropriate use of the chain of command, and evidence-based practice (EBP). However, optimal health and well-being are not guaranteed if care providers fail to advocate for patient safety, fail to communicate with the interdisciplinary team, and fail to invoke the chain of command when it is needed to optimize maternal and fetal health. The lack of competence, adequate staffing, and an organizational culture that does not embrace safety creates a safety hazard and increases the risk of adverse outcomes.

A nurse, physician, or midwife who is simply in and out of the patient's room and who briefly reviews a short part of the fetal/uterine monitor tracing or performs a cervical examination and leaves or who is not fully informed of risks, facts, and changes in the maternal and fetal status creates a safety hazard that increases the risk of adverse outcomes. They will lack a full data set upon which to make clinical decisions. An uninformed care provider cannot provide informed consent. Organizational cultures and care systems will need to change to ensure patient safety and minimize errors (Curtin, 2019; Moeller, Westrate, \& Araujo, 2019).
To maximize safety and reduce the possibility of an adverse outcome, obstetricians need to be present in the hospital during their assigned duty times. They need to analyze the labor curve throughout that time. The presence of an obstetrician, continuous analysis of the labor curve, creation of an individualized plan of care, interdisciplinary collaboration and education, and timely and appropriate actions decreases the incidence of neonatal hypoxic-ischemic encephalopathy (HIE) and death (Tolcher et al., 2016). Competent nurses should also be able to analyze the labor curve in the absence of an obstetrician (Figures 1.1 and 1.2). These competent nurses will individualize the plan of care and inform the obstetric care provider of threats to maternal and fetal safety so that interventions are customized to prevent adverse outcomes.

\section{EVIDENCE-BASED PRACTICE}

EBP is essential to maximize safety and minimize adverse outcomes. EBP needs to be efficient and equitable. Efficient care means waste is avoided when one uses equipment, supplies, ideas, or energy during a treatment or procedure. Equitable care means that the care you provide does not vary in quality because of the patient's gender, ethnicity, country of origin, or socioeconomic status. Evidence-based care means 


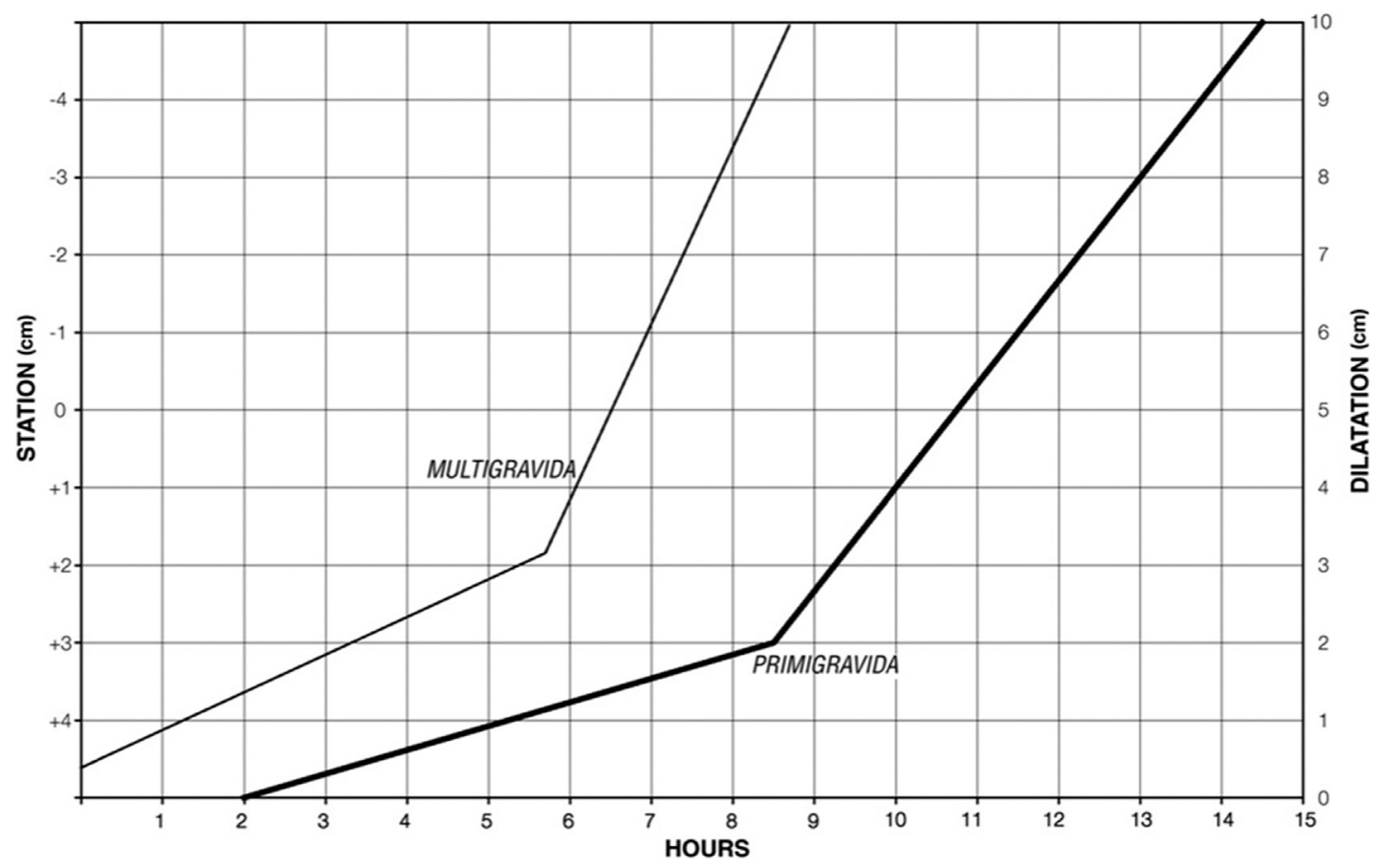

FIGURE 1.1 Example of two partographs or labor curves based on dilatation of the cervix. As can be seen, the top plot is of a multiparous (multigravida) woman and the bottom plot is of a nulliparous primigravida woman.

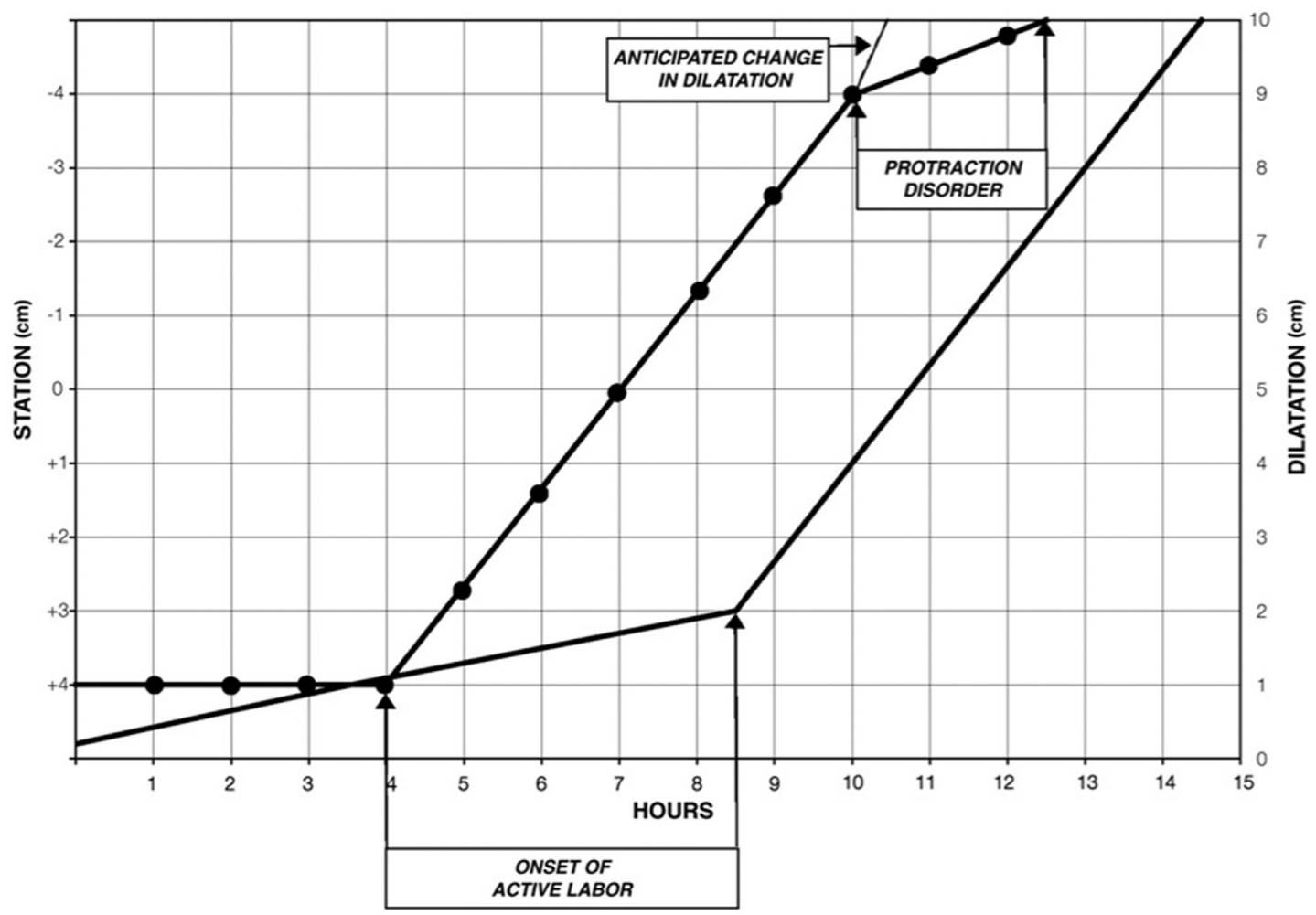

FIGURE 1.2 Labor curve of a nulliparous primigravida woman who was not overweight (bottom line) and labor curve of an obese ( 300 pounds; $136.1 \mathrm{~kg}$ ) nulliparous primigravida woman (top dotted line). Note the different dilatation at the onset of the active phase of labor. Also, note the presence of a deceleration phase that lasted more than 2 hours (protraction disorder) in the woman who was obese.

nurses will have knowledge of credible research findings and will base their practice on research evidence to optimize maternal and fetal outcomes and to promote a woman's sense of safety during the childbirth process. Evidence-based care should be related to research findings that are related to improved outcomes. For example, routine episiotomy was found to be no longer advisable because it contributed to trauma, 
pain, and suffering (Eason \& Feldman, 2000). However, that does not mean a provider cannot apply the unique facts related to the patient. Episiotomy, when needed, does allow faster delivery of the baby's head in some cases.

Childbirth safety also requires competent, unbiased, compassionate, and vigilant care. Care should be based on research evidence and provided by individuals and teams who have the interpersonal and personal characteristics and skills to keep women and their babies safe (Price \& Archbold, 1995).

Physical and emotional safety during labor and birth requires mindful care providers who lack bias and who are not racist. They need to establish a connection with their patients (Lyndon, Malana, Hedli, Sherman, \& Lee, 2018). They will understand the values and wishes of their patients which is part of a just and safe culture. When this occurs, the childbearing woman will be fully informed and an equal partner in a shared decision-making process if an ethical decision-making model is adopted by the healthcare system (Krick \& Chabra, 2018).

Competent nurses will have technical skills and will be able to provide continuous labor support, to intermittently auscultate the fetal heart tones, and to use different technology systems to collect data; they will communicate, exchange and use data in a timely manner; coordinate care; and improve patient safety (Association of Women's Health, Obstetric and Neonatal Nurses [AWHONN], 2018a, 2018b). Competent nurses will work to eliminate preventable harm by engaging in critical thinking. Critical thinking is a clinical judgment process that begins with observation (assessment), followed by reasoning based on knowledge and experience, resulting in clinical judgment. A competent nurse will then be able to foresee the impact of clinical decisions, prioritize patient needs, communicate with other members of the interprofessional team, and coordinate and document patient care to keep patients safe (Gelinas, 2019). They will identify and interpret symptoms, plan care to alleviate complications, and optimize the childbearing woman's physical, psychological, and spiritual health. They will do what it takes to keep their patients safe. Competency of nurses requires specialized knowledge and skills and ongoing education, training, testing, and review of performance. Team training, with nurses, midwives, and physicians, through simulations and/or drills can impact competency development and patient outcomes.

Nurses may not be able to provide competent, evidence-based care if they are overworked and overwhelmed. For example, it was found that the risk of neonatal death was higher on the weekend possibly due to lower hospital staffing and reduced availability of service (Hamilton \& Restrepo, 2003).
Competent nurses will apply the best available evidence from research to optimize maternal, fetal, and newborn health. They will evaluate their own practice behavior and recognize their impact on outcomes (AWHONN, 2018b). They will accept accountability and responsibility for the quality of their practice. They will take ownership of their actions as an individual and as a member of a team. They will work within their nursing scope of practice and will comply with standards and follow evidence-based policies, procedures, and protocols (Sherman \& Cohn, 2019). These nurses will communicate information clearly and accurately under leaders who maximize the activities of team members by ensuring team actions are understood. These leaders will ensure changes in information are shared and necessary resources exist. They will actively scan and assess situations and maintain awareness by monitoring situations. They will anticipate and support team members through accurate knowledge about their responsibilities and workload (Agency for Healthcare Research and Quality [AHRQ], 2013).

\section{BIAS CREATES INEQUITIES}

Bias can create inequities or unequal treatment of childbearing women and result in more maternal deaths or adverse perinatal outcomes (Howell et al., 2018). A competent nurse will provide equitable care and equal treatment that promotes nurturing, inclusion and tolerance, and eliminates bigotry, bias, and discrimination (American Academy of Pediatrics [AAP], 2018). Everyone has biases and therefore purposeful action is needed to acknowledge and eliminate bias during patient care. To eliminate bias, the nurse must recognize and acknowledge what the bias is and be willing to change (Cudé \& Winfrey, 2007).

To keep patients safe, caregivers will demonstrate a lack of racist ideology (Lowe, 2018). They will understand the role of race, ethnicity, language, poverty, literacy, culture, and other social determinants of health. They will work to build a culture of equity within their organization. They will work to ensure a timely and tailored response to patients' needs, especially when inequity or disrespect by other caregivers is observed. They will support shared decision-making and autonomy of the childbearing woman's right to decide how they will be treated when it is appropriate.

Nurses who provide evidence-based and safe care will recognize that their own understanding, actions, and decisions may be based on bias that is unconscious. They may even need cognitive and behavioral 
interventions to reduce bias. These interventions include mindfulness and individuation. Individuation is intentional focus on an individual patient's information in communication with a patient in a way that connects a provider's humanness with the patient's humanness (Howell et al., 2018). Remember the baby is also human and the fetal heart rate pattern is the fetal voice.

\section{COMPASSION}

Competent nurses are compassionate. Compassion is an essential element in quality patient care (Sinclair et al., 2017). Compassion is both cognitive and affective. It is a reflection of one's awareness of another person's distress coupled with a desire to alleviate that distress. It includes affective empathy with emotional resonance, affective understanding of a person's experience, and a person-centered response (Foster, 2009; Sabo, 2006; Sinclair et al., 2017). Just as EBP should be patient centered, compassion must also be patient centered.

Compassion satisfaction occurs when care providers feel connected with their patients and feel a sense of achievement in their work (Hunsaker, Chen, Maughan, \& Heaston, 2015). The compassionate nurse will choose to act to alleviate pain. They will do what they can to provide comfort for their patients and family members. They will imagine what their patients are going through and will react to meet their patients' needs (Bivins, Tierney, \& Seers, 2017).

\section{COMPASSION FATIGUE}

Fatigue is a safety hazard. Fatigue affects skills and communication (The American College of Obstetricians and Gynecologists [ACOG], 2018). Compassion fatigue is a state of exhaustion resulting in physical, emotional, and spiritual depletion and biological, psychological, and social dysfunction as a result of caring for patients in significant emotional pain and physical distress. It is a result of prolonged exposure to compassion stress (Cocker \& Joss, 2016; Lombardo \& Eyre, 2011).

Compassion fatigue is an occupational hazard that occurs when a healthcare worker is exhausted. Fatigued nurses may have decreased alertness, mood changes, decreased energy, decreased ability to concentrate, lack of stamina, lack of motivation, poor judgment, and an inability to learn (Francis, 2018). Nurses with compassion fatigue will have a reduced ability to feel sympathy and empathy. They may be angry and irritable. They will dread working with certain clients or patient and will have a diminished sense of work enjoyment. They may have heightened anxiety and irrational fears or intrusive imagery or dissociation. Nurses who have compassion fatigue will be hypersensitive or insensitive to emotional material. They will have difficulty separating their work life from their personal life. Nurses who have compassion fatigue will experience absenteeism and an impaired ability to make decisions and care for clients or patients. They will have problems with intimacy or in personal relationships and may increase their use of alcohol or drugs. They may demonstrate cynicism at work and have a loss of enjoyment. Some nurses who have compassion fatigue will be depressed or experience stress-related illness (Mathieu, 2007). To diminish the risk or impact of compassion fatigue, become mindful of your feelings, including painful feelings that have pushed you out of your comfort zone. Crying may be an expression of your emotions. Seek out support to share your emotions and focus on the positive. Take care of yourself (Dzubak, 2018).

\section{VIGILANCE}

Competent nurses are vigilant. Vigilance is the cognitive ability to maintain focus and actively and deliberately direct attention to what is believed to be important (Pattyn, Neyt, Henderickx, \& Soetens, 2008). For example, vigilance will include the ability to ignore nonactionable auditory alerts or alarms but to act on actionable alerts and alarms. Vigilance and your attentiveness can be enhanced when you practice being mindful and work to be physically, spiritually, and financially well (Allan, 2018).

Vigilance includes motivation to pay attention to the details and is optimized by a well-rested nurse who sleeps 7 to 8 hours and who takes rest breaks at work. Vigilance can be optimized when clinicians nap at work when possible, but nap less than 45 minutes (which is usually frowned upon in the United States of America; Oken, Salinsky, \& Elsas, 2006; Pattyn et al., 2008; Rogers, 2008). To optimize vigilance, a nap prior to the night shift has been recommended. Other recommendations include exercising when possible; ingestion of caffeinated beverages at the beginning of a shift or about an hour before an anticipated decrease in alertness, for example, between 03:00 and 05:00; and avoidance of fatigue, staffing shortages, and 12-hour shifts (Rogers, 2008).

The vigilant nurse will recognize, act, and verbalize to promote safety. That nurse will know that the fetal heart rate (FHR) pattern changed over time from a normal, reassuring pattern reflective of fetal wellbeing to one with decelerations and/or a decrease in baseline variability with the absence of accelerations. That type of FHR pattern represents potentially hazardous acidemia and it can develop in as little as 1 hour (Paker \& Ikeda, 2007). Vigilance decreases in 
20 to 30 minutes and therefore one cannot "always" or "constantly" watch a computer screen to detect these changes. The paper tracing can be held and shared with other, more rested care providers. Second opinions can be obtained. The nurse who knows the research related to the fetus will know that the fetus can promptly deteriorate and demonstrate deeper decelerations or even bradycardia. They will not think, "Oh that's just a little common deceleration" or "I'm reassured because I still have some variability." The competent, well-informed nurse will think "Wow, the fetus needs better cerebral blood flow and oxygenation. There is only one blood vessel that brings blood and oxygen to the fetus (the umbilical vein). I need to act now and communicate these changes to the provider."

\section{VIGILANCE FATIGUE}

Vigilance fatigue is also known as vigilance decrement (Pattyn et al., 2008). Vigilance fatigue impacts decisions and is similar to intoxication with slowed reaction times or an increase in errors, especially during tedious monitoring tasks. The impact of vigilance fatigue can include information processing deficits, poor decision-making, impaired performance, and clinical errors (Rogers, 2008). Care providers who are experiencing vigilance fatigue will have slowed reaction times, may disengage from a task, or may experience attention withdrawal (Körber, Cingel, Zimmermann, \& Bengler, 2015; Pattyn et al., 2008). Researchers found impaired vigilance begins to occur after 20 to 30 minutes of time on a task, which can result in a failure to detect critical changes (Pattyn et al., 2008). Reduced vigilance may precede clinical errors and injury to the mother or her baby (Körber et al., 2015; Oken et al., 2006; Rogers, 2008).

\section{FATIGUE AND FAULTY COMMUNICATION}

Successful decision makers and communicators are well rested, not driven by pressures, and aware of their biases. To eliminate bias, you must recognize and acknowledge what the bias is and be willing to change (Cudé \& Winfrey, 2007). Being well rested may be a difficult goal, but it is an important goal that prevents errors and patient injuries. Research shows that error rates increase when nurses work more than 12 hours a day, or more than 40 hours a week (Rogers, Hwang, Scott, Aiken, \& Dinges, 2004). Unfortunately, nurses often work more than 40 hours a week. Some nurses work more than 6 days in a row and many rotate shifts (Trinkoff, Geiger-Brown, Brady, Lipscomb, \& Muntaner, 2006). If you do the same, you increase your risk of becoming overtired, chronically stressed, or injured-and you therefore increase the risk of injuring a patient. For example, sleep-deprived military gunners hit their targets but shot at the wrong target (Kushida, 2005). An error-prone environment is one that has inadequate staff to get the job done, rude coworkers or supervisors, insufficient resources, everchanging technology, too little training, excessive paperwork, and communication failures (Welker-Hood, 2006). If you work in an error-prone environment, share your concerns with nursing management.

Clinical reasoning, good problem-solving, sound judgment, and effective clinical decision-making require clear thinking, knowledge, and experience (Croskerry, 2003, 2005, 2006). Experience will help you develop your clinical reasoning. Good clinical reasoning means inductive, fast, and intuitive recognition of a problem. Some people call this "having a gut feeling." However, it is based on knowledge and experience, not just a feeling. For example, when a pregnant woman presents with decreased fetal movement, frequent mild contractions, a non-reassuring fetal heart rate pattern, a closed cervix, and blood in her shoes (known by skilled nurses as "the positive shoe sign"), an experienced nurse will think there is a placental abruption. The obstetrician will be called and the patient will be prepared for surgery.

Other decisions are a result of a slower, rational, deductive, rule-based, analytical process. This process takes more time, but is valuable because it results in fewer errors (Croskerry, 2006). Errors in our decisions increase when we are uncertain, hurried, pressured, or have biases (Croskerry, 2003). Therefore, if you are new to the practice of labor and delivery nursing and/or you feel something is amiss, or you feel hurried but lack the confidence, knowledge, or motivation to make a decision or to call the midwife or physician, it is best to admit you need help and confer with a more experienced nurse or your charge nurse. By constantly expanding your experiences and knowledge base, you should become a better decision maker and communicator, and you will increase your ability to see the broad range of possibilities in any given clinical situation.

\section{ADVOCACY}

Advocacy requires a nurse to recognize, verbalize, and mobilize. Advocacy requires the ability to make evidence-based decisions that are patient centered. Decisions about patient care should be based on knowledge of maternal and fetal risk factors, research, current assessments, changes in the maternal or fetal status following interventions, and input from the patient and her family. You must be able to face 
problems head on and not delay decisions until it is too late. Do you know the current plan of care the patient desires (birth plan)? Do you know the plan the provider desires? If not, ask the midwife or physician, for example, "What is the plan of care for Ms. Iaminlabor?" Individualize the plan of care and educate the childbearing client on what is probable, possible, and not possible.

The individualized plan of care should be in the best interest of your patient and her unborn baby. If there is no clear plan of care, or if you cannot accept it, discuss your concerns with the charge nurse and midwife or physician. The plan of care must be patient centered and must prevent harm. If you believe a decision is needed to deliver by cesarean section or to expedite delivery with forceps or a vacuum extractor (an operative vaginal delivery), communicate with the charge nurse or your supervisor first. Discuss the clinical facts that support a change in the plan of care. If you do not have a charge nurse or supervisor, speak directly with the physician or midwife. It is far better to be proactive and to help the midwife or physician make a patient-centered decision now than to wait for them to arrive at the bedside later and make a decision at the last moment when the fetus is decompensating.

\section{COMMUNICATION EXAMPLE: Staff nurse to} charge nurse communication example: "I have been seeing variable decelerations that are getting deeper and longer but the baby is still at 0 station with caput and molding. We have been pushing for 2 hours. I am concerned about the need for a cesarean section. I know the pregnancy weight gain was 50 pounds and she is 5 feet tall. I'm concerned the baby is just not going to fit. Can you discuss my concerns with the physician? I do not want to start an oxytocin infusion because cephalopelvic disproportion is a contraindication plus she has enough contractions and we are pushing. I don't think more uterine power will help. I think she needs a cesarean section.”

Advocacy requires support of a reasonable plan of care, anticipation of potential problems, and knowledge of what is normal and abnormal. You should know what the fetal heart rate baseline was on admission. Is the current fetal heart rate baseline rising or falling? A rising baseline may be a fetal catecholamine (stress) response to hypoxia. Falling baselines are usually a fetal decompensation response. Both are abnormal.

Advocacy requires a nurse to mobilize the operating room crew to expedite delivery before fetal decompensation, neurologic injury, or death. However, this is a rare event. Examples of acute events where the fetus quickly decompensates include a ruptured vasa previa when an amniotomy is performed, a uterine rupture, or an amniotic fluid embolism. In those cases, it will be obvious that delivery needs to be expedited. Inform the charge nurse of actions that have been taken and the current maternal and fetal status to ensure a safe and expeditious delivery. When cesarean sections are not performed on your labor and delivery unit, you will need to inform the operating room crew through your house supervisor that you need to mobilize them if the pattern continues or worsens. Ask the physician to come to the bedside. If there is fetal tachycardia, especially with decelerations or minimal or absent variability and no accelerations, the fetus is in jeopardy. Initiate intrauterine resuscitation measures. Communicate to mobilize resources. If you have no charge nurse, ask another nurse to prepare the operating room and mobilize the crew while you call the physician to come to the bedside. Call the anesthesia provider and the neonatal team so that they are on their way to the hospital. You will need to be proactive and request the presence of the obstetrician at the bedside any time you see tachycardia, bradycardia, or decelerations that last more than 30 seconds, especially if they are becoming deeper and longer. Communication among the healthcare team members is imperative to help ensure the individuals required to provide safe care to the mother and baby or babies are available in a timely manner.

\section{COMMUNICATION}

Within the subculture of labor and delivery are the people with whom you may need to communicate. They may be difficult to communicate with if they are know-it-alls, passives, dictators, "yes" people, "no" people, or gripers (see Table 1.1).

\section{Nurse-to-Patient Communication}

Patients may also take the role of know-it-all, passive, dictator, griper, or someone who always agrees with you or quickly disagrees with you. On the other hand, they may be open, honest, and genuinely interested in what you have to say. An assessment of their interaction with other family members will help you recognize their communication style and characteristics. Communication with patients, family members, and other healthcare providers is essential to help you prevent adverse events (Joint Commission on Accreditation of Healthcare Organizations, 2004). Communication also creates relationships. Therefore, you will need to find a way to relate to your patient and her family and friends to maximize the effectiveness of your communications. By developing your 


\begin{tabular}{|c|c|}
\hline Personality Type & Characteristics and Suggestions for Communication \\
\hline Know-it-alls & $\begin{array}{l}\text { Arrogant, usually have a strong opinion on every issue. When they're wrong, they get defensive. Vali- } \\
\text { date their ideas. Tell them you see their point of view (if you can), then ask for their help in solving a } \\
\text { problem. }\end{array}$ \\
\hline Passives & $\begin{array}{l}\text { Never offer ideas and never let you know where they stand. For example, they may perform a vaginal ex- } \\
\text { amination and then walk out of the room and not tell you their findings. Follow them out of the room. } \\
\text { Establish eye contact. Ask them to tell you their findings. }\end{array}$ \\
\hline Dictators & $\begin{array}{l}\text { Bully and intimidate. They're constantly demanding and brutally critical. Don't try to be their friend. Just } \\
\text { be specific in what you would like them to do and ask them if they can help you. If they refuse, ask } \\
\text { your charge nurse or nursing supervisor to intervene or help you. }\end{array}$ \\
\hline "Yes" people & $\begin{array}{l}\text { Agree to cover your patient while you are on lunch break and then never see your patient. When you re- } \\
\text { turn from your break and see they have not charted their observations, ask them to do so. If they do } \\
\text { not comply, notify your charge nurse or supervisor of their failure to see your patient. Document in the } \\
\text { record the time you expected them to see your patient and their name. }\end{array}$ \\
\hline "No" people & $\begin{array}{l}\text { Quickly point out why something won't work and are inflexible. Avoid these people unless you have to } \\
\text { interact with them. Keep your communications brief and clear. }\end{array}$ \\
\hline Gripers & All they do is complain. See all of the above. \\
\hline
\end{tabular}

emotional intelligence, you should be able to recognize your feelings and the feelings of others, and then regulate your personal feelings and expressions in response to clinical situations (Stichler, 2006).

Your first nurse-patient interaction is critical in establishing a trusting relationship with your patient and her family and friends. Introduce yourself by stating your name and licensure, and state that you will be her nurse for the next specified number of hours. When your patient speaks to you, face her, listen closely, and paraphrase what you hear. Make eye contact when it is culturally accepted, do not interrupt her, and do not multitask. It is extremely rude to look at a chart or a computer screen instead of your patient.

COMMUNICATION EXAMPLE: "Hi, Ms. Jones. I'm Michelle and I'll be your nurse today. I'm here for the next 12 hours. I understand from Gayle, your night nurse, that you are $4-\mathrm{cm}$ dilated, that your plan is to have intravenous medication later in the labor, and that your husband wants to cut the cord after the birth. Is that correct? Good. It looks like you are contracting every 3 minutes and they feel strong to me. Your baby looks good. I see there are accelerations in the heart rate. Is there anything you need right now?”

Create an environment that is conducive to open communication and low stress to enhance a feeling of trust. You can enhance the environment by limiting the number of visitors and other noise and distractions (Stichler, 2007a). Sit at eye level with your patient when you communicate. Pick your words wisely, as words might upset patients and families. For example, "failure to progress" or "noncompliant cervix" are demeaning and disparaging (Katz, 2005).

You should use open-ended questions to assess your patient's knowledge of the labor and delivery process. For example, you might say, "What questions do you have about what is going to happen today?" Some patients believe they have no questions because they have been watching birthing programs on television. They will tell you they know everything they need to know about their epidermal. In spite of their misconceptions, it is important for you to discover their knowledge deficits and provide information during teachable moments. For example, prior to rupture of the membranes, you might want to mention that the sac around the baby has no nerves. One patient was terrified because she heard the nurse say, "The doctor will be rupturing your brains." Another patient reported she had condominiums "down there." She really had condylomata (warts). Try not to laugh. Discover the source of your patient's anxiety and fear. Perhaps her sister, cousin, or mother had a traumatic birth experience and she fears the same thing will happen to her. You will need to reassure her that you are there for her support and safety and will be checking in frequently during her labor. Use humor sparingly and appropriately. Listen carefully to her complaints and concerns because you may be the 
first person she tells or the first person who recognizes there is a problem.

To facilitate open communication, acknowledge the partner and/or labor coach and any family members present in the room. Ask them their names and recognize their supporting roles. Be sure the primary coach and the father of the baby eat and rest if there is a long labor ahead. The father-to-be may wish to play an active role during the labor process or no role at all. Assess their needs and desires as well as your patient's needs and desires. Satisfaction with the support of their partner results in less patient stress, less depression, and less anxiety for as long as 3 months after delivery (McVeigh, 1997).

Support family-centered care, if that is desired, by keeping the baby in the birthing room after delivery. Support skin-to-skin contact between the mother and her baby. If she chooses to breastfeed, you should support breastfeeding within the first hour of the baby's life (Phillips, 2003). Communicate with the patient to learn her expectations for labor and birth.

If there is a written birth plan, read it and acknowledge its contents. Sometimes desires or plans are more like wishes that you may not be able to fulfill. You will need courage to do the right thing for your patient's safety and health, even when it seems undesired or unpopular or goes against her birth plan or wishes.

CLINICAL EXAMPLE: A nulliparous woman in labor was four feet six inches tall. At about 5 -cm dilation, her membranes spontaneously ruptured. There was dark green, particulate meconium in the amniotic fluid. Variable decelerations appeared on the tracing. The midwife found there was a face presentation. The physician was called into the room and decided that a cesarean section was necessary. When the patient was informed, she was sad because she had planned for a vaginal delivery. The nurse said, "I'm sorry but the baby didn't read the plan." The patient said, "Neither did my sister's baby." The nurse asked for clarification. The patient told her that her sister was also petite like her and had a baby with a face presentation. In this case, there was a good outcome for both the mother and her baby.

Nurses who communicate to discover and respond to patient needs prevent injuries (Kendig, 2006). To protect patients and prevent injury, communication should be purposeful and goal oriented. For example, there may be people in the room who are watching television and talking among themselves yet the nurse perceives the laboring woman needs a quiet room because she is preeclamptic, hyperreflexive, and hypertensive. In this case, the nurse would explain the patient's needs and ask the visitors to leave or limit their conversation. The hospital's visitor policy should provide the framework for information shared with the people in the room.

ACOG (2011) believes that actively involving patients in their care will increase diagnostic accuracy, patient satisfaction, and adherence to therapy, thus resulting in improved health. They recommend that healthcare providers (a) speak slowly and use plain, nonmedical language; (b) limit the amount of information provided; (c) repeat information; (d) use teach-back or show-me techniques; (e) create an atmosphere in which patients can ask questions; and (f) provide written materials to reinforce oral explanations. Naturally, if the patient does not speak English, a certified interpreter should be located. Record the name of the certified interpreter or translation service in the medical record.

\section{Nurse-to-Nurse Communication and Nurse-to-Provider Communication}

Communication between team members is vital to ensure patient safety and the continuity of care based on an individualized care plan. When you receive the change of shift or handoff report, you must know if the amniotic fluid was clear but now has meconium in it. You must know the baby's normal baseline level, what it is now, and time of the last acceleration (a reflection of normal $\mathrm{pH}$ ). The last acceleration should have occurred within the last 90 minutes, even if narcotics were administered. You must know how to interpret the tracing, and over time you will become more comfortable with its physiologic meaning.

The nurse is the conduit through which information flows to the midwife and/or physician. It has been said that the nurse is the "eyes and ears" of the midwife and physician. You are the one who keeps the charge nurse informed of changes. You have the power to make a difference. Therefore, it is your duty to advocate for the physician's presence at the bedside when the parents have requested it. You have a duty to update them at reasonable intervals so that they stay abreast of the maternal and fetal condition throughout the labor process.

\section{SBARR}

The letters S, B, A, R, and R represent the words situation, background, assessment, recommendation, and response. The situation is the patient's current condition and your major concern. The background includes the pertinent facts from the patient's history. The assessment is what you think the problem is. The recommendation is what you recommend the provider 
do. The response is what the provider said or did when you made your recommendation (Cherouny, Federico, Haraden, Leavitt Gullo, \& Resar, 2005; Guise \& Lowe, 2006; Nunes \& McFerran, 2005).

To help midwives and physicians make good decisions, they need you to provide them with relevant facts, abnormal findings or laboratory results, and any other information that paints a complete picture in their minds. For example, if there is fetal tachycardia, you will also want to inform them of the maternal pulse and temperature. Both are elevated when there is chorioamnionitis. If there is vaginal bleeding, they will need to know about contractions and details related to pain, such as the location of the pain, whether it is constant or intermittent, sharp or dull, how the patient is responding to the pain, and the fetal status. If you need a physician or midwife at the bedside, you might say "I need you to come to the hospital now" or "The patient (fetus or mother) needs you to come to the bedside now." When the midwife and/or physician respond to your request without delay, they promote a safe, reliable organization that is patient centered.

DOCUMENTATION EXAMPLE: Jane Doe, CNM, notified at 2330 of patient with BP 156/92, facial and hand edema, pitting ankle edema. Normal vision, not blurred and sees no flashes of light. Hyperreflexive with no clonus. Complains of unrelenting headache and heartburn. No vaginal bleeding. Contractions every 1 to 2 minutes lasting 30 to 40 seconds with less than 1 minute between contractions. Patient reported no history of preeclampsia. Recommended CNM come to bedside immediately. CNM informed this nurse she was on her way. Recommended this nurse call obstetrician to come to the bedside immediately. CNM agreed.

The change of shift report should be comprehensive (see Box 1.1). By the end of the report you should know events that happened during the last shift, changes in the mother's or baby's status, and the interdisciplinary plan of care. You will need to decide if the current plan of care is safe and reasonable for your patient. If not, request a change in the plan of care after you report your findings and share your concerns.

COMMUNICATION EXAMPLE: Requesting a change in the plan of care: "Dr. Iamallears, may I speak with you a moment about the current plan of care? I just learned in report that your patient, Jane Doe, now has a fever. Her temperature is 100.8 degrees. In addition, her pulse is 116 beats per minute. She's been dilated 5 centimeters for the last 3 hours. There's caput at 0 station. There are variable decelerations that are getting deeper. I just discontinued the Pitocin infusion that was at $30 \mathrm{mU} /$ minute. Would you please come and evaluate her and let me know if you'd like to make any changes in the current plan of care?"

If a medication is due at the time of change of shift, for example 7 p.m. or 19:00, the departing nurse should administer that drug and inform the arriving nurse that the medication was given. It is also important to report the patient's intake and output, and her dietary restrictions.

Physical or psychological needs that promote patient well-being should be communicated to midwives and physicians (Henrikson, 2006). Nurses should

BOX 1.1 Elements of a complete nurse-to-nurse report

1. Patient's name and name of her partner or guardian, patient's age, gravida, parity, due date or weeks of gestation, number of fetuses, and reason for admission or diagnoses, height and current weight.

2. Provider's name, location, and telephone number.

3. Allergies, including allergy to latex, and Group B Streptococcus status.

4. History of transfusion reactions or prenatal laboratory results that might affect care during labor or birth.

5. Current medications and past medications if they affect or potentially affect her current condition or care, e.g., antidepressants after her last delivery.

6. Maternal habits, e.g., alcohol, tobacco, or street drug use.

7. Prenatal/obstetric and medical history.

8. Last vital signs and any abnormal findings.

9. Physical assessment of abnormal findings.

10. Fetal normal baseline rate (based on past nonstress tests and/or the admission report).

11. Current fetal baseline rate and other features of the fetal heart rate pattern.

12. Current uterine activity.

13. Last cervical examination, fetal station, status of membranes, including color, amount, and odor of amniotic fluid.

14. A review of progress using the plotted labor curve of dilatation and descent, especially when there was been a protraction or arrest of dilatation and/or descent.

15. Last dose of medications, including prostaglandins, oxytocin, antibiotics, narcotics, insulin, antihypertensives, etc.

16. Current pain level and ability to cope with the labor, and patient desires for pain management. 
also communicate to clarify orders, discuss therapeutic plans, report changes in the patient's condition, share questions the patient or her family members have, and report abnormal or significant findings.

Strong nurse-physician relationships affect both nurse and patient satisfaction. If nurses and physicians have equal power within their respective practice areas and they collaborate to provide patient care, staff nurses will continue to work at that hospital. In fact, increased communication between nurses and physicians reduces bad outcomes (McClure \& Hinshaw, 2002). Communication and relating to others requires a sense of social competency (Stichler, 2007b). Social competence includes the ability to assess the emotions of others and relate to them in a manner that diffuses anger and conflict, provides encouragement, or inspires them. Social management is part of emotional intelligence or being intelligent in our relationships (Goleman, Boyatzis, \& McKee, 2002).

If you work with certified nurse midwives, you should work to develop a positive relationship with them. It will help if you know that they probably are limited in what they can do without the consultation of the backup obstetrician (see Boxes 1.2-1.4).

Nurses must notify the midwife or physician when an order should not be followed. The American Medical Association (AMA) supports communication between a nurse and a physician when the nurse finds an order to be in error or contrary to customary medical or nursing practice. The AMA has opined that "the physician has an ethical obligation to hear the nurse's concern and explain those orders to the nurse involved. The ethical physician should neither expect nor insist that nurses follow orders contrary to standards of good medical and nursing practice" (AMA, 1997).

If you encounter a clinical situation in which you believe a physician's opinion, attention, or care is

BOX 1.2 Certified nurse midwife management without a requirement for a physician consultation

1. Gestational diabetes (diet controlled).

2. Fetus that is 36 or more weeks of gestation.

3. Internal and external fetal monitoring, including insertion of a fetal spiral electrode and intrauterine pressure catheter.

4. Meconium with reassuring fetal heart rate pattern.

5. Group B Streptococcus prophylaxis according to protocol.

6. Urinary tract infection diagnosis and treatment.

7. Initiation of anesthesia request when a normal spontaneous vaginal delivery is expected.

8. Amnioinfusion.

9. Episiotomy.

10. First- and second-degree laceration repair. needed, discuss this with the midwife. If the midwife refuses to communicate with the consulting physician and you still feel a physician's care is needed for your patient, share your concern with the midwife in the presence of a member of your chain of command such as the charge nurse or house supervisor. The chain of command is now a chain of resolution to solve a problem. The charge nurse or supervisor should possess the authority and responsibility to act in the best interests of the patient. If that nursing leader also finds that a physician is needed, it is their responsibility to discuss the need for a physician evaluation with the midwife and they may even call the physician if the midwife refuses to do so or fails to do so.

To improve your communication abilities, develop your emotional intelligence. Stay calm when people try to provoke you. In the event you work with a "know-it-all" who hangs up the telephone or walks away from you while you are speaking or before you have finished communicating, or before you make

BOX 1.3 Examples of certified nurse midwife intrapartal management that may require a physician consultation (consult your hospital's requirements)

1. Grand multiparity or great grand multiparity

2. Severe anemia (hemoglobin $<9 \mathrm{mg} / \mathrm{dL}$ )

3. Estimated fetal weight $>4500 \mathrm{~g}$

4. Postterm pregnancy (gestation of 42 weeks or more)

5. Pregnancy $<36$ weeks completed weeks of gestation

6. Induction of labor

7. Augmentation of labor

8. Maternal fever with or without suspected chorioamnionitis

9. Preeclampsia

10. Thick and/or particulate meconium-stained amniotic fluid

11. Regional anesthesia in the presence of dystocia (protracted or arrested dilatation and/or descent)

12. Arrest of dilatation or descent

13. Second stage $>2$ hours especially when there was a protraction of dilatation during the first stage of labor, a protraction or arrest of descent, or any suspicion of cephalopelvic disproportion

14. Fetal heart rate pattern that requires intrauterine resuscitation (tachycardia or bradycardia; absent or minimal variability; variable, late, prolonged and/or spontaneous decelerations; few to no accelerations)

15. Vaginal bleeding

16. Conditions that may require physician intervention: Fetal malposition, compound presentation, or the need for the application of forceps, a vacuum extractor, or a cesarean section 
BOX 1.4 Examples of midwife and physician intrapartal collaborative management (consult your hospital's requirements).

A physician should come to the bedside:

1. Insulin-dependent diabetic or not well-controlled gestational diabetic without suspected fetal macrosomia

2. Known intrauterine growth restriction

3. Fetal heart rate patterns that require intrauterine resuscitation but do not improve with these interventions:

a. Discontinuation of the oxytocin infusion

b. Position change to a lateral position to optimize cardiac output

c. Correction of hypotension (e.g., administration of ephedrine 5-10 mg slow IV push)

d. Hyperoxygenation (using a tight-fitting simple face mask at 10 or $15 \mathrm{~L} /$ minute for a nonrebreather mask with an oxygen reservoir)

e. Stop pushing or push with every other contraction

f. Tocolysis (e.g., IV bolus or terbutaline $0.25 \mathrm{mg}$ SQ or slow IV push)

4. Preeclampsia with "mild" laboratory abnormalities

5. Excessive bleeding during labor

6. Fetal malpresentation or malposition

7. Greater than 30 minutes during the third stage of labor (delivery to placenta time)

8. Protracted or arrested descent during the second stage of labor

9. Vaginal birth after cesarean section

10. Excessive uterine tenderness or rigidity

11. Suppression of preterm contractions

12. Sickle cell anemia or disease

a reasonable request for your patient, do not chart "Provider hung up on me" or "Provider walked away as I was speaking." Instead, notify your nursing leader in your chain of command of the situation and explain your patient's needs that are not being addressed by the provider. Ask your nursing leader to make the next call. This action is a form of advocacy. It is invoking the chain of command that has also been called the chain of communication or chain of resolution. A chain of resolution describes what staff members and leaders are doing to resolve issues and concerns regarding patient care and safety. It may also address resolving issues related to environmental needs, equipment, or other things (Hardie, 2012). If there is no charge nurse or nursing leader, ask another nurse to listen on another telephone line (if possible) and call the "know-it-all" again. In the second call, communicate that your colleague is listening in. Your colleague should say, "hello." Then you should ask the provider again for what your patient needs. If the provider hangs up or refuses to come in, activate the chain of command and record who you notified in the chain.

\section{CHAIN OF COMMAND}

Each hospital should have a chain of command policy, procedure, or protocol. These have also been called the Chain of Command or Chain of Resolution policies, procedures, or protocols. You may need to activate your chain of command to resolve conflicts over patient management plans (Mahley \& Beerman, 1998). The chain of command is a process that is used when the nurse feels that ethical or practice standards are not being maintained or there are unresolved conflicts or clinical issues that affect patient well-being. Prior to invoking the chain of command, every effort should be made to clearly and fully communicate with the midwife, resident, or physician directly involved in the patient's care. If the communication fails, the chain of command policy must be followed.

Unresolved conflicts often involve a disagreement in patient care, such as the need to apply oxygen or a spiral electrode. The chain of command or chain of communication is invoked as the patient's safety net. Your role as a patient advocate creates an ethical duty to prevent harm, and requires courage to acknowledge the disagreement and seek its resolution. You might say to the midwife or physician (away from the patient's bedside), "We have an unresolved disagreement here. I've asked the charge nurse to help us resolve this issue." You can also say, "I'm activating our chain of command policy." The charge nurse may decide to assume care of your patient or assign another nurse to care for your patient. The charge nurse can be asked to speak with the midwife and may need to speak with the next highest level in the chain, that is, the midwife's backup physician or the attending physician. If the provider is the Chief of Obstetrics, involve your nursing chain of command, for example, the charge nurse and nurse manager. If the Chief of Obstetrics is also the Chief of the Medical Staff, involve your charge nurse or supervisor, who may need to involve the Vice Chief of the Medical Staff to resolve the conflict.

Documentation of the chain of command should include the observations and events that created a need to use the chain of command, specific facts, and the time of events and communications.

DOCUMENTATION EXAMPLE: Dr. Knowitall ordered oxytocin. Physician was informed at nurses' station at 1205 that patient was contracting every 2 minutes $\times 50$ to 70 seconds, moderate to palpation. Order to administer 
oxytocin was questioned. Charge nurse Sally Smith informed of communication with physician. Nurse Smith spoke with physician who insisted on oxytocin administration. Chief of $\mathrm{OB}$ Dr. Iknowmore was called by charge nurse at 1210. Nurse Manager Tanya Doitright updated by Sally Smith.

The nurse's responsibility and duty is to advocate for the patient's safety. It is imperative that the nurse continue to communicate with the charge nurse or supervisor until the conflict is resolved. The nurse must act to prevent injury. Patients really want a nurse who cares for them as if they were a family member. They want a nurse who is responsive to their needs, including their physical, emotional, and spiritual needs. They want a nurse who is willing to do extra things and who follows through on promises (Trossman, 2007). Sometimes our communications are ineffective in moving toward a patient-centered goal. For example, what would you do if the physician asked you to do something in front of the patient that you felt was not best for the patient or her baby?

If you receive a verbal order at the bedside, but are concerned that following the order may harm the patient, do not follow the order. Before the provider leaves the room, repeat the order to clarify their intent and understanding of the facts. For example, an order you should question is "Begin oxytocin per protocol on this patient" but you know the patient has a baby in a transverse lie. When the provider leaves the room, follow him or her. In the hallway ask if he or she wanted oxytocin to be administered to this patient who has a transverse lie. If the answer is yes, do not follow the order. Instead, speak to your charge nurse or supervisor and inform them of the order, the provider's response, and your concerns. The charge nurse or supervisor has the responsibility and authority to speak with the provider so that the order is changed. Your responsibility is to continue to care for your patient.

In your career, there will be times when you will need to initiate the chain of command and involve your charge nurse or house supervisor-or even the Chief of Obstetrics. If you have a clinical nurse specialist, they often work with the Patient Care Manager as a dyad and may also be able to help you obtain the care you need for your patient. In some cases, the Director of Maternity Services or Maternal Child Services, the Assistant Vice President for Women and Children's Services, or the Executive Vice President for Patient Care Services will be called. When the Chief Nursing Officer is called, the entire nursing chain of command will be involved.

You can develop your own list of names and phone numbers of people in your chain of command. It is a good idea for the hospital to give you a copy of the chain of command policy. It is your responsibility to find it if you do not receive it. From time to time you may want to return to the policy for guidance or to refresh your memory. In general, there are two "chains" of command that compose the overall chain of command: the nursing chain of command and the medical chain of command (see Figures 1.1 and 1.2).

Activate the chain of command or chain of communication whenever there are unresolved issues related to patient care or if you have concerns related to a provider (Figures 1.3 and 1.4). If resolution of the conflict

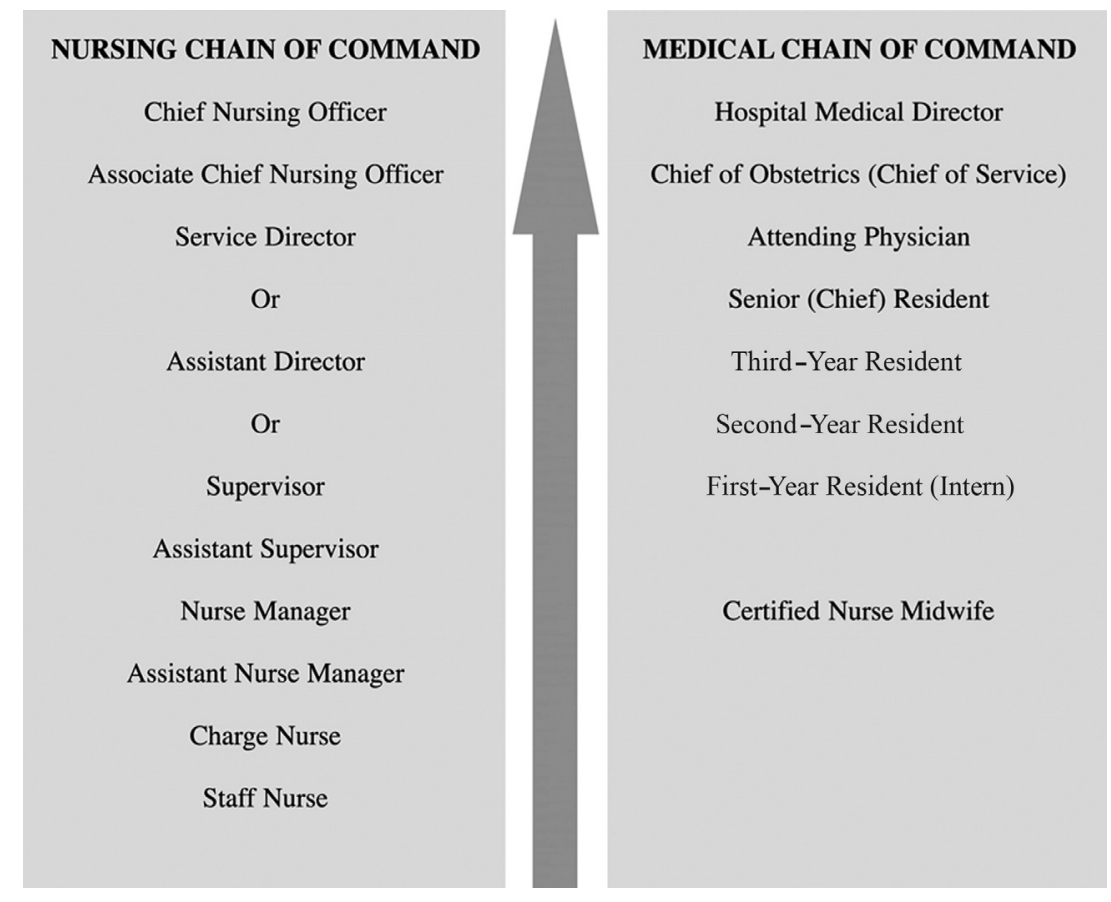

FIGURE 1.3 Sample chain of command with the staff nurse as the first link in the chain. 


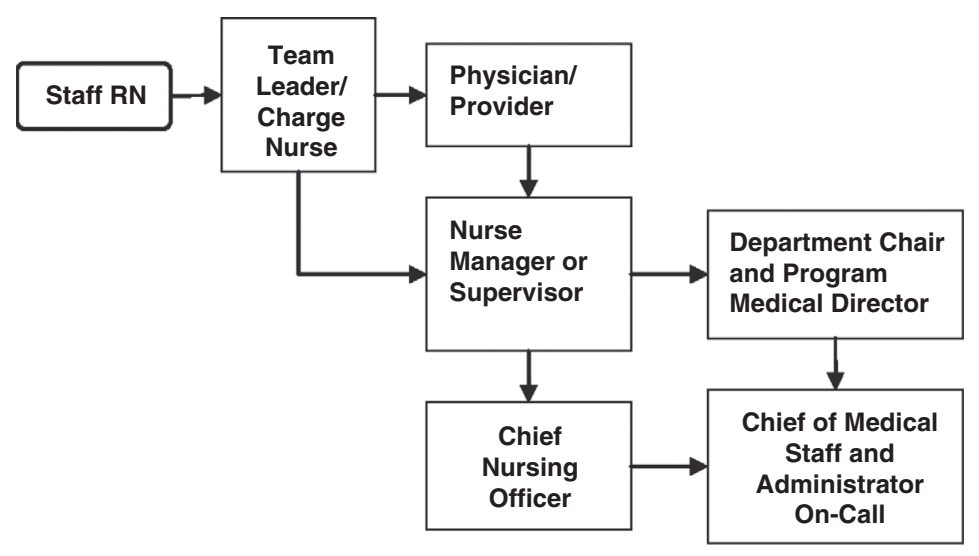

Reasons to Activate the Chain of Command or Chain of Communication

1. Issues requiring communication.

2. Issues surrounding patient care.

3. Concerns related to physican/provider.

FIGURE 1.4 Example of the chain of command, chain of communication, chain of resolution with the staff nurse as the first link in the chain.

or issue is not achieved at any step up the chain of command, continue up the chain until a mutual resolution is achieved. During this process, the childbearing woman must still receive care to prevent an adverse outcome. Perhaps only once in your entire career will you follow the chain all the way to the top (Chief Nursing Officer and Hospital Medical Director).

If discussions with the midwife or physician do not result in appropriate care, the nurse is responsible to ensure timely and appropriate actions (Simpson \& Chez, 2001). Advocacy for the patient may require you to invoke your hospital's chain of command.

A retrospective review of 90 risk manager files from 1995 to 2001 revealed that adverse outcomes are directly related to procedures and people (White, Pichert, Bledsoe, Irwin, \& Entman, 2005). Half of those files were related to labor and delivery; the rest were related to gynecologic surgery $(38 \%)$ and ambulatory care $(12 \%)$. Communication failures were associated with $31 \%$ of the adverse events. For example, there were disruptions in the flow of critical information from one caregiver to another, or there were communications that upset the patient or her family.

EXAMPLE: Failure to invoke the chain of command resulting in an adverse outcome: Dina was multiparous. She arrived in labor and delivery with a thick, closed cervix and a full-term fetus at a -3 (floating) station. Within 6 hours she was dilated $5 \mathrm{~cm}$ but there was an arrest at $5 \mathrm{~cm}$ for another 3 hours. During that 3 hours, the FHR pattern deteriorated and lacked any sign of fetal well-being. Intrauterine resuscitation measures were undertaken without improvement in the FHR. The attending family medicine physician was called and a telephone order to begin an oxytocin infusion was received.

Instead of withholding the oxytocin, the nurse administered it. Within 30 minutes there were late decelerations. By that time, the attending physician was present and the nurse shared the tracing and pointed out the late decelerations. The physician insisted the oxytocin infusion be resumed.

Instead of withholding the oxytocin infusion and using the chain of command to obtain the care of an obstetrician, the nurse administered oxytocin again. However, the nurse did inform the attending family medicine physician that the on-call obstetrician would be asked to come to the bedside for a second opinion.

The on-call obstetrician arrived; ordered the oxytocin infusion to be discontinued and it was. Both physicians remained on the labor and delivery unit but no cesarean section was ordered.

Three hours later, Dina's cervix dilated to an anterior lip but the fetal heart rate became bradycardic with late and prolonged decelerations. The nurse never informed another nurse or a nursing leader of this emergency. The nurse never advocated for a cesarean section.

One hour later, the cervix was completely dilated and the attending physician applied a vacuum extractor in Dina's labor room. There was no double set up in an operating room. The baby's head was delivered but there was 
a shoulder dystocia and the baby could not be delivered vaginally. A Zavanelli maneuver was performed and Dina was moved to an operating room for a cesarean section. Apgar scores were 0 at 1 minute, 3 at 5 minutes, and 3 at 10 minutes. The newborn weighed 4,345 grams, was asphyxiated, and brain damaged.

Dystocia is derived from the Greek word for "abnormal" or "difficult" and the Greek word tokos for "labor" or "delivery." A difficult delivery of the shoulders was encountered in this example. In fact, all maneuvers failed to deliver the baby. Shoulder dystocia could have been avoided had oxytocin not been used to force the fetus down into the pelvis, the nursing chain of command had been activated when Dina's baby was bradycardic but remote from delivery, and an obstetrician had ordered and performed an emergency cesarean section.

The plan of care should be more than "monitor the patient" or "expect vaginal delivery." It should be individualized and provide a road map toward a safe outcome. Sometimes the plan of care is in conflict with the patient's needs. To resolve clinical issues related to patient care conflicts, your hospital should have a "Resolution of Clinical Issues" or "Resolution of Conflict" procedure. This procedure should define the channels of communication and decision-making that you should follow when there are questions or concerns regarding medical or nursing care. Knowing your patient should be the basis for clinical decisions and judgments and individualized care.

When orders deviate from the plan of care or customary or safe practices, discuss the situation and your concerns with the midwife and/or physician(s) responsible for the patient. If the issue needing clarification is not resolved, the chain of command should be initiated and your charge nurse or immediate supervisor must be notified. The charge nurse or supervisor will discuss the concern with the midwife or physician. If the issue remains unresolved, other individuals in the "chain of command" will become involved so that the conflict can be resolved. Avoid documentation that reflects a disagreement between yourself and others. Instead, document your assessments, plans, actions, evaluations, and communications.

EXAMPLE: Inappropriate documentatio reflecting disagreement between a nurse and a physician: Dr. Disreguarde aware of lack of fetal well-being throughout second stage of labor. Dr. desires no interventions at this time. Nurse manager in room. Dr. orders pushing to continue. Dr. informed of meconium and lack of fetal well-being. Dr. asked if pediatrician and neonatal nurse should be called for cesarean section. Dr. said no. Dr. reminded of particulate meconium and lack of fetal well-being. Dr. said there is no meconium.
EXAMPLE: Appropriate documentation reflecting disagreement between a nurse and a physician: Frequent discussions with Dr. Disreguarde during second stage about FHR pattern, including meconium and lack of fetal well-being at this time. No new orders. Practice coordinator (charge nurse) in the room at this time speaking with physician. Nurse manager called the pediatrician, anesthesia provider, and nursery nurse to attend delivery.

The first narrative demonstrates the lack of collaboration and teamwork. The second demonstrates how well the chain of command worked to secure personnel for delivery.

\section{DISRUPTIVE BEHAVIOR}

Disruptive behavior hinders effective communication. Disruptive behavior is personal conduct, whether verbal or physical, that affects or potentially may affect patient care negatively (Lazoritz \& Carlson, 2008). Disruptive behavior includes intimidation, violence, inappropriate language or comments, sexual harassment, and/or inappropriate responses to patient or staff requests. Hanging up midsentence or refusing to come in are examples of disruptive behavior.

Nurses must not participate in disruptive behavior and should report it to their leader(s). Disruptive behavior diminishes the capacity of all team members to provide safe care. If someone verbally threatens you, that is disruptive behavior. Throwing things such as instruments or lap sponges in your direction is disruptive behavior. No person with whom you work should ever hurt you. Abuse and battery must not be tolerated-and must always be reported. If you feel you are the victim of disruptive behavior in the workplace, speak with your nurse manager and discuss the incident. Disruptive behavior cannot and must not be tolerated and silence on your part is not an option. Speak to the "disrupter" privately with your supervisor present. In the meeting, state the disruptive behavior by explaining what you found to be disruptive. Keep your statement brief, factual, and descriptive. Also share how you felt when the disruptive behavior occurred. When confronted, the disrupter may argue or yell or dispute what you say. If this happens, politely thank the disrupter for their time and leave. Write an occurrence or problem/resolution report and include the disrupter's response. Continue to act professionally, even if the disrupter is rude to you. If the behavior continues, follow the same plan: Meet, discuss, report. The report provides an opportunity for the disrupter to change their behavior (Lazoritz \& Carlson, 2008).

Advocating for patient safety can be difficult when a physician or any other care provider is sleep 
deprived and/or fatigued. This impacts performance. The fatigued and/or sleep-deprived care provider may demonstrate a change in their normally pleasant mood. They may not retain information, may have slower performance than usual, and may not communicate effectively which can result in errors that hurt patients (ACOG, 2018).

A family member may be disruptive either from fatigue or from a belief system. For example, a husband may refuse to let his wife have a cesarean section because he is concerned it will mutilate her body. Empathy is needed to defuse disruptive behavior. Try to understand these concerns and inform the physician. Your responsibility is to deescalate the situation to maximize patient safety. Remember the family's stress and that they may act out for many reasons. Work from the onset of the first meeting to establish rapport. Listen carefully to what they say and what is not said, and look for a theme. Observe their body language. Do not raise your voice. You may say, "I'm here to keep your wife (loved one) safe" or "We're on the same team and we can work together." Do not think of it as "us versus them" but all as important members of the healthcare team. Offer explanations; be realistic; pay attention to your behavior; avoid anger, closed body language, and aggression. Stay calm; keep a relaxed posture, and if culturally acceptable, make eye contact. Keep your hands visible. You also may want to ask another staff member to be with you (Jubb \& Baack, 2019).

\section{EXPECTED AND UNEXPECTED COMMUNICATION WITH THE PROVIDER}

Obstetric care providers expect four basic types of telephone calls (see Box 1.5). If you are trying to decide whether to call the physician or midwife, just make the call. The time of day should not stop you from sharing important patient information. Call when the patient needs a medication, is not tolerant of labor, is ready to push, or any time there is concern for the fetus. Call for orders, to clarify orders, or to change orders.

BOX 1.5 Telephone calls from a nurse to a physician or midwife

1. Patient has arrived

2. Status update and request for new orders

3. New, significant findings and a need to come to the unit to assess the patient

4. There is an emergency and we're taking the patient to the operating room for a cesarean section
When there are new and/or significant findings, it is especially important that you report information in a timely manner (meaning within a few minutes of knowing the information) to the midwife or physician. For example, if your patient complains of constant pain in the suprapubic area and her bladder is empty, think about the fit of the fetal head in the pelvis. Is it pressing down above her pubic bone? Ask the midwife or physician to come to the bedside and evaluate this unusual pain. If you are given an order, be sure you accept that it is safe to follow that order. If you are concerned about a risk of harm, discuss it with the person who wrote the order. It is your responsibility to question orders that may harm the patient and you must not follow harmful orders.

Communicate and collaborate with the primary healthcare provider to ensure there is an appropriate individualized plan of care and evaluation of the patient's condition prior to beginning a procedure such as cervical ripening or induction. For example, you might say to the midwife or obstetrician, "I'm really worried about the baby. Can you please come in and evaluate the tracing for me?" If you do not get the response you desire, it may be because you did not communicate your degree of concern. State clearly "I am concerned. I would like you to come to the bedside now. I need you to evaluate (tell them what you need)." If you still do not get the response you desire, use your chain of command process and inform the provider "I am using the chain of command (communication or resolution) because I need a provider at the bedside now." Alternatively, you can ask "I understand you are not available to come to the bedside now. Who can I call in your place?" You might ask, "Do you have remote viewing so that you can review the tracing immediately?" Then, document that you asked they review the tracing remotely and their response to your request.

\section{ORGANIZATIONAL CULTURES}

Advocacy, communication, the chain of command, and evidence-based practice occur within organizations that have an identifiable culture. The healthcare organizational culture includes norms, attitudes, values, assumptions, customs, and behaviors (Chervenak \& McCullough, 2005; Lefton, 2007; Seren \& Baykal, 2007).

\section{A Culture of Safety}

A high-reliability organization (HRO) has a culture of safety where prevention of adverse events is an obvious system-wide goal. Within that type of organization, nurses will not have barriers to report adverse events. Adverse events will be investigated and 
root causes that resulted in injury or death will be identified. Changes in policies or procedures may be enacted to improve or optimize safety and prevent adverse outcomes.

A healthy organization has a culture that is committed to honest business practices and is focused on the needs of patients, community, and society (Chervenak \& McCollough, 2005). Great leaders create an environment that enables the best solutions and the best decisions (Henrikson, 2007). In a healthy organization, communication should occur among the patient, her partner, her friends and visitors, and all members of the healthcare team (Simkin \& Ancheta, 2005).

\section{Collaborative or Competitive Organizations}

Healthcare organizations may also be collaborative or competitive. In a collaborative organization, there is teamwork and team management to reach a desired goal. In a competitive organization, achievement, a sense of superiority, excellence, and possessing technology that is better than other organizations will be the goals. If the organization has a power culture, authority will be the center of attention, and tasks will be assigned by a manager.

\section{Cynical Organizations}

Cynical organizations have leaders who are not supportive and who create a hostile work environment. Communication in that type of organization can be difficult or even intimidating. An unresponsive culture will have leaders with a dictatorial, top-down, threatening, and punishing style of behavior. This type of leadership creates communication barriers. A submissive culture will be unresponsive, will have minimal expectations and communication, and will provide minimal feedback. A submissive, but responsive, culture will have people who always agree with you, who talk a lot but do not listen, who gloss over performance issues, who only provide positive feedback, and who seek harmony (Lefton, 2007). It is important that you determine the type of organization and culture within which you work.

\section{A Culture of Civility}

In a culture of civility, disruptive behavior is less likely to occur. Leaders will be transformational. Transformational leaders model behavior; encourage the heart; inspire a shared vision; enable others to act; and challenge the process. They support open communication, inspiration, enthusiasm, and support positive change. They empower others through shared decision-making (Clavelle \& PradoInzerillo, 2018).

In a culture of civility, patient needs are the number one priority. Nursing leaders support nurses and do not tolerate or perpetuate workplace incivility. To promote respect and civility, these leaders will socialize their prospective employees to inform them that they expect civility and a respectful atmosphere; they will conduct unit meetings to share civility norms with new hires and current staff; they will create and post staffing guidelines to ensure safe patient care and a healthy work environment; and they will address uncivil behavior and hold employees accountable for their behavior (Smith, 2019).

\section{A Toxic Culture}

The opposite of a toxic culture is a culture of civility. A toxic culture includes staff who gossip and who express negativity. The toxic culture has high absenteeism and turnover, bullying and incivility. Bullies are cruel, uncaring, uncivil, and brutal to their colleagues (Thompson, 2019). If you experience incivility, report it to your leader(s) so that your concerns can be addressed in an ethical and fair manner. However, if you work in a toxic culture, your concerns may never be addressed and you may need to consider leaving that environment and working elsewhere.

In a toxic culture, new staff will not receive a warm welcome. There is a distrust of leaders, a lack of transparency, and a failure to manage unmotivated staff. There is no accountability for professional practice in a toxic culture (Sherman, 2019). You may experience moral distress where you believe you know the right thing to do but feel unable to pursue that course of action due to constraints (Whitehead, Herbertson, Hamric, Epstein, \& Fisher, 2015). In a toxic culture, there is a sense of a lack of psychological safety. Staff do not feel safe to speak their minds. Errors will be unreported and mistakes will be made. There is a lack of teamwork (Sherman, 2019). Clearly, a toxic culture is unsafe and patients can be injured.

\section{LABOR AND DELIVERY MODEL OF CARE}

Within the dominant healthcare organization culture there are subcultures. For example, the culture in postpartum care may be different from the culture in the nursery or in labor and delivery. The norms, attitudes, values, assumptions, customs, and behaviors within the subculture of labor and delivery affect the quality and quantity of communication (Tucker et al., 2006). Communication is also influenced by the design of the care model. The four types of 
intrapartal services include the nurse-managed labor model, the academic/teaching model, the nurse-toattending physician communication-on-site model, and the nurse-to-nurse midwife communication-onsite model (Simpson, 2005). For example, if you are the primary patient care provider and the midwife or physician is not in the building, you work within a nurse-managed labor model. In that case you are responsible for recognizing problems, evaluating labor progress, providing hands-on care, and informing other team members when they are needed.

In the nurse-to-nurse midwife or physician communication-on-site model, it should be easy to communicate with the provider. However, even if the provider is in the hospital, they may be distracted by the needs of other patients. Since the labor nurse, midwife, and physician are part of one collaborative team, be sure to keep them up to date so that they do not miss an opportunity to make clinical decisions that promote patient safety. If you need them to come to the bedside, ask them to do so.

DOCUMENTATION EXAMPLE: Spontaneous rupture of membranes, fluid clear, nonfoul, saturated $1 / 3$ of linen protector. Variable decelerations noted. Certified Nurse Midwife (CNM) called on postpartum unit and informed of spontaneous rupture of membranes, clear fluid, variable decelerations. Requested CNM come to bedside immediately to evaluate patient. CNM stated she was on her way.

Perhaps you work with residents in an academic/ teaching model setting. In this setting, the residents will assess the cervix and fetal station and insert internal monitors such as the spiral electrode or intrauterine pressure catheter. Nurse-to-resident communication may be hindered by the resident's need to control the decision-making process. However, that does not mean you should withhold your findings or concerns. If you feel the plan of care needs to change, you must speak up.

Ineffective or inadequate communication is a threat to patient safety. In fact, communication issues were found to be the primary root cause that resulted in injury during delivery and infant death after delivery (The Joint Commission, 2004). Other root causes included organization culture as a barrier to effective communication and teamwork, hierarchy and intimidation, failure to function as a team, and failure to follow the chain of communication. Other root causes identified by The Joint Commission (2004) were staff competency, orientation and training, inadequate fetal monitoring, unavailable monitoring equipment and/or drugs (resources), unavailable or delayed physician, and unavailability of prenatal information.
COMMUNICATION CLINICAL EXAMPLE:

You observe late decelerations and no accelerations, and the resident orders you to administer oxytocin. You change your patient's position to her right side, place a tight-fitting nonrebreather face mask with oxygen flowing at $15 \mathrm{~L} /$ minute, adjust the ultrasound transducer and tocotransducer, and note the continuation of late decelerations. You also note her blood pressure is normal. You say, "I'd like to wait at least 15 minutes to see if the baby's heart rate improves." The resident dons a sterile glove, rubs the fetal scalp, evokes an acceleration, and insists you administer the oxytocin immediately. You then say, "There have been no spontaneous accelerations for the last hour, variability is absent or minimal, and the late decelerations continue to persist in spite of the intrauterine resuscitation actions." You then call your team leader or charge nurse to the room using the patient call light. At that point, if the resident continues to insist on the administration of oxytocin, the charge nurse and the resident should leave the room to continue the discussion about the plan of care.

\section{CARE COORDINATION}

Care coordination ensures patient's needs and preferences for health services and their health information is shared with people at various sites over time (McCammon \& Francis, 2018). Optimal safety requires care coordination and continuity of care with a lack of chaos or rapid change. Feeling safe also depends on a patient's sense of connection and respect from care providers. The organization also needs excellent communication between and among members of the healthcare team (Lyndon et al., 2018). The organization must lack bias and demonstrate its cultural values and policies in order to reduce racial inequities. There must be a lack of structural racism, systemic racism, or institutional racism. There should be a culture of respect where actions are taken toward others that protect, preserve, and enhance their dignity. The organizational culture must be accountable and include leaders that optimize the balance between the individual and the system (a just and safe culture). The just and safe culture will have a robust reporting system where employees feel confident that the contents of their report will remain confidential and lead to system improvements not punishment (Howell et al., 2018).

Unlike a just and safe culture, a culture of perfection will blame employees for mistakes, and shame individuals. This will result in a decrease in the number of reports of errors (Zabari \& Southern, 2018). A 
high-reliability organization (HRO) is another name for a just and safe culture. An HRO will have systems in place to reduce the risk of error and support error reporting. An HRO is a just, safe, and civil organization that encourages error reporting. It will have leaders who have a preoccupation with failure and who work to make things right. These HRO leaders will be aware of internal and external factors in the team, technology, and environment; will possess a questioning attitude and challenge assumptions; will bounce back from mistakes before there is more damage; and will identify experts or those with the most experience in a situation (Moeller et al., 2019). There may be interdisciplinary team huddles every 4 hours during labor for FHR tracing review, which can improve term fetal and neonatal health outcomes (Thompson, Krening, \& Parrett, 2018). Policies, procedures, and protocols will be evidence based with research and other references. Other safety huddles may be developed that include review of all of the patients (board reports); individualized patient huddles (shift change reports); and/or spontaneous huddles where the nurse calls together the interdisciplinary team.

In any organization, women in labor have an increased risk of HIE of their baby. Evidence-based practice can reduce the incidence of HIE. For example, it was found that implementation of a labor and delivery safety bundle, especially for women who had a high-risk pregnancy, reduced the risk of fetal brain damage and neonatal HIE. In a study of outcomes for 5,826 deliveries, researchers found fewer adverse outcomes for women who had a high-risk pregnancy. There was a reduction in uterine rupture, third- and fourth-degree lacerations, HIE, and birth trauma. The safety bundle that resulted in fewer adverse outcomes required the obstetrician to physically be present at all times during their scheduled duty shift and required they review the labor curve (partogram) to maintain situational awareness. Also, obstetricians were informed of adverse outcomes as part of their annual staff evaluation. In addition, as a result of obstetrician presence and review of the labor curve, there was a significant reduction in liability claims and payments (Tolcher et al., 2016). If the obstetrician is not present, there will be a lack of oversight by the attending obstetrician and there may be ineffective communication among team members (a finding of Tolcher et al. [2016] that was a root cause of HIE of a neonate). Using this evidence from Tolcher et al. (2016), prevention of adverse outcomes in the absence of an obstetrician will require a competent nurse who reviews and analyzes the labor curve and who promptly reports abnormalities to the obstetrician. The nurse must have the knowledge to properly interpret the labor curve and the fetal heart rate and uterine activity tracing. The nurse must have the foresight and courage to request the obstetrician's presence at the bedside to prevent adverse outcomes in high-risk women. They must be willing to say, "I am concerned. I am uncomfortable. This is a safety issue" (AHRQ, 2013).

\section{PROVIDER ROLES AND EXPECTATIONS}

In order to know whom to call or with whom to share information, you need to know the roles and responsibilities of the other healthcare providers. Your hospital should have policies and procedures that define the maternal/child services for family practitioners, residents, obstetricians, and anesthesia providers. For example, family practitioners may be credentialed to evaluate the condition of the mother and infant, order medications, deliver the infant when there is a cephalic presentation (or by using low forceps or a vacuum extractor), and resuscitate the infant. They may be credentialed to repair the episiotomy, but must consult with the obstetrician for certain conditions. An obstetrical consult may be required for some procedures. An obstetrician may need to be consulted for abnormal bleeding, a retained placenta, preeclampsia, prolonged labor, multiple gestation, induction of labor, polyhydramnios, before any obstetrical operation or breech delivery, for medical or surgical complications, for preterm labor and tocolysis, or for a trial of labor after a cesarean section.

Obstetricians perform duties similar to family physicians, but they also usually have privileges for midforceps and cesarean sections. Anesthesia providers evaluate the condition of the mother prior to the administration of an anesthetic agent, place and remove indwelling epidural catheters, inject medication into the epidural catheter, initiate continuous infusions administered through the epidural catheter, and remain immediately available during the induction of epidural anesthesia. They rarely know how to interpret the fetal heart rate pattern or uterine activity pattern, and you should not expect them to do so. Therefore, it is your responsibility to be sure the maternal and fetal conditions are stable prior to the administration of an anesthetic. If the mother or fetus is unstable, speak to the obstetric care provider prior to the administration of analgesia or anesthesia.

Physicians and/or certified nurse midwives may create the initial plan of care with informed consent of the patient. The nurse is responsible for coordinating care, suggesting changes in the plan of care, and knowing who else is on the team taking care of the patient. An error can be committed if the wrong plan is followed or there is a failure to complete a planned action (Institute of Medicine, 1999). Therefore, you 


\section{TABLE 1.2 GENERIC NURSING PLAN OF CARE ELEMENTS}

\begin{tabular}{|c|c|l|}
\hline \multirow{2}{*}{1.} & Problem: & Alteration in self-perception related to anxiety. \\
\cline { 2 - 4 } & Outcome: & Patient will understand procedures and processes and adapt without undue additional anxiety. \\
\hline \multirow{2}{*}{2.} & Problem: & Alteration in comfort related to progress in labor and delivery. \\
\cline { 2 - 4 } & Outcome: & Patient will become comfortable. \\
\hline \multirow{2}{*}{3.} & Problem: & Potential for infection related to rupture of membranes. \\
\cline { 2 - 4 } & Outcome: & Patient will be free of infection. \\
\hline \multirow{2}{*}{4.} & Problem: & $\begin{array}{l}\text { Alteration in maternal and/or fetal perfusion and oxygen delivery related to contractions and labor } \\
\text { process. }\end{array}$ \\
\cline { 2 - 4 } & Outcome: & Patient will progress through labor and delivery without complications. \\
\hline \multirow{2}{*}{5.} & Problem: & Alteration in self-perception related to expanded role. \\
\cline { 2 - 4 } & Outcome: & Patient will experience time to bond with her infant after delivery. \\
\hline \multirow{2}{*}{6.} & Problem: & Potential for hemorrhage following delivery related to altered hemodynamics. \\
\cline { 2 - 4 } & Outcome: & Patient will recover without unusual blood loss. \\
\hline
\end{tabular}

must know the plan of care and communicate with other healthcare team members often during your shift to accomplish that plan of care or to change the plan of care. As the patient's condition changes, the plan of care must also change.

\section{AN INDIVIDUALIZED EVIDENCE-BASED PLAN OF CARE}

A generic nursing plan of care is not individualized and may not even be evidence based. However, both should include a nursing diagnosis. Some nursing diagnoses were approved by the North American Nursing Diagnosis Association International (NANDA). For example, labor pain is a NANDA-approved nursing diagnosis. Your hospital may have a list of other nursing diagnoses; you might also create a nursing diagnosis. Nursing diagnoses are the problem or potential problems (see Table 1.2).

\section{CHANGING THE INDIVIDUALIZED PLAN OF CARE}

EBP that is patient centered should optimize health and reduce the risk of harm to childbearing women and their babies. Nurses communicate with providers when they have assessed evidence, formed a nursing diagnosis, and realize the plan of care needs to change to optimize maternal and fetal health and to prevent harm. Nurses also identify care issues that need ethical, legal, or risk intervention. Once the nurse realizes the plan of care needs to change, communication with the nursing leader or provider (in the absence of a nursing leader such as a charge nurse) is required. Advocacy for a change in the plan of care may be needed if the nursing leader or provider is unresponsive to the nurse's request. If communication with a provider intimidates you, talk to your charge nurse or a more experienced nurse and ask for help communicating to change the plan of care. Once you have communicated patient data and/ or their request(s), document it in the medical record. Include the time you communicated, to whom you spoke, what you said, what that person or persons said, and any actions taken by you, your supervisor, or the provider.

DOCUMENTATION EXAMPLE: Subtle late decelerations, fetal heart rate baseline sinusoidal-like. Dr. Seinwave called at home. Informed of baseline 130 to $140 \mathrm{bpm}$, recurrent subtle late decelerations, contractions every $2 \mathrm{~min}$ utes lasting 50 seconds, moderate to palpation. Telephone order for oxytocin received and read back to physician. This nurse did not act on the oxytocin order. This nurse did request the physician to come to bedside now to evaluate the fetal-pelvic fit and the fetal heart rate and contractions. Charge Nurse Ima Responsible notified of the above. Patient notified the physician was called regarding concerns. 
DOCUMENTATION EXAMPLE: Recurrent variable decelerations with baseline $140 \mathrm{bpm}$, minimal variability. Dr. Listo called to the bedside and is en route. OB Hospitalist called to the bedside. This nurse reviewed tracing with $\mathrm{OB}$ Hospitalist Dr. Aqui. Cesarean section ordered and clinical care coordinator (charge nurse) notified of urgent cesarean section order.

Nurses recognize, verbalize, and mobilize. "In emergencies, when prompt action is necessary and the physician is not immediately available, a nurse may be justified in acting contrary to the physician's standing orders for the safety of the patient" (AMA, 1997). Staffing should be based on the acuity of patients and standardized protocols should be followed for emergencies (Fariello \& Paul, 2005). Therefore, if you recognize a problem, notify other nurses, and tell them what you want them to do. Remain calm, maintain a patient safety focus. A pilot once said, "In a turbulent storm, just keep the wings level. Tough it out. Fall apart after you land."

\section{CONCLUSIONS}

For women and their children to be safe, healthcare providers must have compassion, be vigilant, and practice evidence-based care within a healthcare system that has a culture of safety. Effective, closed loop communication is necessary to provide the best care for patients and to prevent errors and injury (Cherouny et al., 2005). Cognitive errors with a lack of critical thinking and appropriate decisions can occur and do occur and result in poor communication and documentation. Cognitive errors can and do result in the lack of individualized plans of care, needed actions, injury, and death of patients. To optimize outcomes and maximize safety, find a place of work that has a healthy culture. Determine if it is an HRO with safety as a priority and open and collaborative communication among team members. If concerns continue or issues related to care arise, own your ethical duty to be a patient advocate. Consult members of your chain of command/communication/resolution as you see a need. You are a vital part of the healthcare team. You are responsible for the safety and wellbeing of the people you care for.

\section{REVIEW QUESTIONS}

True/False: Decide if the following statements are true or false.

1. Childbirth safety requires competent, compassionate and vigilant care but bias is not a factor.
2. An exhausted healthcare worker may have compassion fatigue.

3. Attention to detail (vigilance) can decrease in 20 to 30 minutes resulting in vigilance fatigue, a safety hazard.

4. One element of a complete nurse-to-nurse report at the beside is a review of the labor curve and any abnormalities.

5. A time to communicate with a midwife or physician is when the patient has a physical or psychological need.

6. Patients can provide valuable information to increase the ability of physicians to diagnose their condition.

7. A toxic culture has high absenteeism, incivility, bullying, but a low turnover of personnel.

8. Chain of command is invoked as a nurse's ethical duty as a patient advocate to promote patient safety and prevent harm.

9. The last $\mathrm{R}$ in SBARR stands for the patient's response to interventions.

10. Patients want a nurse who follows the physician's orders even when they have the potential to harm the patient.

\section{REFERENCES}

Agency for Healthcare Research and Quality. (2013). TeamSTEPPS® 2.0 pocket guide (AHRQ Pub. No. 14-0001-2). Rockville, MD: Author.

Allan, S. H. (2018). Nurse perception of alarm fatigue impacts compliance with alarm management. Education can help nursing staff gain control and improve patient safety. American Nurse Today, 13(5), $26-28$.

American Academy of Pediatrics. (2018). AAP diversity and inclusion statement. Pediatrics, 141(4), e20180193. doi:10.1542/peds.2018-01777

American College of Obstetricians and Gynecologists. (2011). Partnering with patients to improve patient safety (Committee Opinion Number 490). Washington, DC: Author.

American College of Obstetricians and Gynecologists. (2018). Fatigue and patient safety (Committee Opinion Number 730). Washington, DC: Author.

American Medical Association. (1997). Code of medical ethics: Current opinions and annotations. Chicago, IL: Author.

Association of Women's Health, Obstetric and Neonatal Nurses. (2018a). Health information technology for the perinatal setting. Washington, DC: Author.

Association of Women's Health, Obstetric and Neonatal Nurses. (2018b). Standards for professional nursing practice in the care of women and newborns (8th ed.). Washington, DC: Author.

Bivins, R., Tierney, S., \& Seers, K. (2017). Compassionate care: Not easy, not free, not only nurses. BMJ Quality \& Safety, 26, 1023-1026. doi:10.1136/ bmjqs-2017-007005

Cherouny, P. H., Federico, F. A., Haraden, C., Leavitt Gullo, S., \& Resar, R. (2005). Idealized design 
of perinatal care. (IHI Innovation Series White Paper) Cambridge, MA: Institute for Healthcare Improvement.

Chervenak, F. A., \& McCullough, L. B. (2005). The diagnosis and management of progressive dysfunction of health care organizations. Obstetrics \& Gynecology, 105(4), 882-887. doi:10.1097/01.AOG.0000156302 $.37189 .2 \mathrm{f}$

Clavelle, J. T., \& Prado-Inzerillo, M. (2018). Inspire others through transformational leadership. American Nurse Today, 13(11), 39-41.

Cocker, F., \& Joss, N. (2016). Compassion fatigue among healthcare, emergency and community service workers: A systematic review. International Journal of Environmental Research and Public Health, 13, 618. doi:10.2290/ijerph13060618

Croskerry, P. (2003). Cognitive forcing strategies in clinical decision-making. Annals of Emergency Medicine, 41(1), 110-120. doi:10.1067/mem.2003.22

Croskerry, P. (2005). The theory and practice of clinical decision-making. Canadian Journal of Anesthesia, 52, R1. doi:10.10.1007/BF03023077

Croskerry, P. (2006). Critical thinking and decision-making: Avoid the perils of thin-slicing. Annals of Emergency Medicine, 48, 720-722. doi:10.1016/j.annemergmed.2006.03.030

Cudé, G., \& Winfrey, K. (2007). The hidden barrier. Gender bias: Fact or fiction? Nursing for Women's Health, 11(3), 255-265. doi:10.1111/j.1751-486X.2007.00165.x

Curtin, L. (2019). Errors, care, and the bottom line. American Nurse Today, 14(2), 44.

Dzubak, J. L. (2018). Nurses cry, too: What happens when we can't fix everyone. Strategies for processing a patient's death. American Nurse Today, 13(6), 50-51.

Eason, E., \& Feldman, P. (2000). Much ado about a little cut: Is episiotomy worthwhile? Obstetrics \& Gynecology, 95(4), 616-618. doi:10.1097/00006250 -200004000-00027

Fariello, J. Y., \& Paul, E. (2005). Patient safety issues in a tertiary care hospital's labor and delivery unit. Nursing for Women's Health, 9(4), 321-323. doi:10.1177/1091592305280876

Forster, D. (2009). Rethinking compassion fatigue as moral stress. Journal of Ethics inMental Health, 4(1), $1-4$.

Francis, R. (2018). Nurse fatigue: A shared responsibility. American Nurse Today, 13(11), 26.

Gelinas, L. (2019). Nursing care: The quest to eliminate preventable harm. American Nurse Today, 14(2), 4.

Goleman, S., Boyatzis, R., \& McKee, A. (2002). Primal leadership: Realizing the power of emotional intelligence. Boston, MA: Harvard Business School Publishing.

Guise, J. M., \& Lowe, N. K. (2006). Do you speak SBAR? Journal of Obstetric, Gynecologic, and Neonatal Nursing, 35(3), 313-314. doi:10.1111/j.1552-6909.2006.00043.x

Hamilton, P., \& Restrepo, E. (2003). Weekend birth and higher neonatal mortality: A problem of patient acuity or quality of care? Journal of Obstetric, Gynecologic, and Neonatal Nursing, 32(6), 724-733.

Hardie, R. (2012). Chain of resolution: A bold new way to go. Journal of Obstetric, Gynecologic, and Neonatal Nursing, 41, S98. doi:10.1111/j.1552-6909.2012 $.01361 . c$
Henrikson, M. (2006). Great leaders are made, not born. AWHONN Lifelines, 10, 335-338. doi:10.1111/ j.1552-6356.2006.00064.x

Henrikson, M. (2007). Great leaders are made, not born: Conclusion of a four-part series. AWHONN Lifelines, 10(6), 510-515. doi:10.1111/j.1552-6356.2006 $.00101 . \mathrm{x}$

Howell, E. A., Brown, H., Brumley, J., Bryant, A. S., Caughey, A. B., Cornell, A. M., ... Grobman,W.A. (2018). Reduction of peripartum racial and ethnic disparities: A conceptual framework and maternal safety consensus bundle. Journal of Obstetric, Gynecologic, and Neonatal Nursing, 47(3), 275-289. doi:10.1016/j.jogn.2018.03.044

Hunsaker, S., Chen, H.-C., Maughan, D., \& Heaston, S. (2015). Factors that influence the development of compassion fatigue, burnout, and compassion satisfaction in emergency department nurses. Journal of Nursing Scholarship, 47(2), 186-194. doi:10.1111/ jnu. 12122

Institute of Medicine. (1999). To err is human: Building a safer health system. Washington, DC: National Academies Press.

The Joint Commission. (2004). Sentinel event alert \#30. Oakbrook Terrace, IL: Author.

Joint Commission on Accreditation of Healthcare Organizations. (2004). Preventing infant death during delivery. (Sentinel Event Alert No. 30) Oak Brook, IL: Author.

Jubb, J. M., \& Baack, C. J. (2019). Verbal de-escalation for clinical practice safety. American Nurse Today, 14(1), 5-7.

Katz, A. (2005). Think before you speak. AWHONN Lifelines, 9(2), 105-106. doi:10.1111/j.1552-6356 .2005.tb00760.x

Kendig, S. (2006). Primary care safety. AWHONN Lifelines, 10(6), 502-509. doi:10.1111/j.1552-6356 .2006.00100.x

Körber, M., Cingel, A., Zimmermann, M., \& Bengler, K. (2015). Vigilance decrement and passive fatigue caused by monotony in automated driving. Procedia Manufacturing, 3, 2403-2409. doi:10.1016/ j.promfg.2015.07.499

Krick, J., \& Cjabra. S. (2018). On "end-of-life decision making for parents of extremely preterm infants". Journal of Obstetric, Gynecologic, and Neonatal Nursing, 47, 415-417. doi:10.1016/j.jogn.2018 .01 .007

Kushida, C. A. (2005). Sleep deprivation: Clinical issues, pharmacology, and sleep loss effects. New York, NY: Marcel Dekker.

Lazoritz, S., \& Carlson, P. J. (2008). Don't tolerate disruptive physician behavior: Bad behavior harms both staff and patients. Reporting it benefits everyone. American Nurse Today, 3(3), 20-22.

Lefton, C. (2007). Does your workplace culture need CPR? American Nurse Today, 32-35.

Lombardo, B., \& Eyre, C. (2011). Compassion fatigue: A nurses' primer. The Online Journal of Issues in Nursing, 16(1), 3. doi:10.3912/OJIN .Vol16No01Man03

Lowe, N. K. (2018). Disparities in the health of women and children. Journal of Obstetric, Gynecologic, and Neonatal Nursing, 47, 273-274. doi:10.1016/j.jogn .2018.04.001

Lyndon, A., Malana, J., Hedli, L. C., Sherman, J., \& Lee, H. C. (2018). Thematic analysis of women's 
perspectives on the meaning of safety during hospitalbased birth. Journal of Obstetric, Gynecologic, and Neonatal Nursing, 47, 324-332. doi:10.1016/ j.jogn.2018.02.008

Mahley, S., \& Beerman, J. (1998). Following the chain of command in an obstetric setting: A nurse's responsibility. Journal of Legal Nurse Consulting, 9(1), 7-13.

Mathieu, F. (2007). Running on empty: Compassion fatigue in health professionals. Rehabilitation and Community Care Medicine, 4, 8-10. Retrieved from http://www.compassionfatigue.org

McCammon, S., \& Francis, R. (2018). Moving forward with nurse-led care coordination. American Nurse Today, 13(5), 23.

McClure, M. L., \& Hinshaw, A. S. (Eds.). (2002). Magnet hospitals revisited: Attraction and retention of professional nurses. Washington, DC: American Nurses Association.

McVeigh, C. (1997). Motherhood experiences from the perspective of first-time mothers. Clinical Nursing Research, 6(4), 335-348. doi:10.1177/ 105477389700600404

Moeller, D., Westrate, R., \& Araujo, M. (2019). Safety culture: A journey to zero. American Nurse Today, 14(2), 44-46.

Nunes, J., \& McFerran, S. (2005). Perinatal patient safety project. Permanente Journal, 9(2).

Oken, B. S., Salinsky, M. C., \& Elsas, S. M. (2006). Vigilance, alertness, or sustained attention: Physiological basis and measurement. Clinical Neurophysiology,117(9), 1885-1901. doi:10.1016/ j.clinph.2006.01.017

Parer, J. T., \& Ikeda, T. (2007). A framework for standardized management of intrapartum fetal heart rate patterns. American Journal of Obstetrics and Gynecology, 197(1), 26.e1-26.e6. doi:10.1016/ j.ajog.2007.03.037

Pattyn, N., Neyt, X., Henderickx, D., \& Soetens, E. (2008). Psychophysiological investigation of vigilance decrement: Boredom or cognitive fatigue? Physiology\& Behavior, 93, 369-378. doi:10.1016/ j.physbeh.2007.09.016

Phillips, C. (2003). Family-centered maternity care. Sudbury, MA: Jones and Bartlett.

Price, V., \& Archbold, J. (1995). Development and application of social learning theory. British Journal of Nursing, 4(21), 1263-1268. doi:10.12968/ bjon.1995.4.21.1263

Rogers, A. E. (2008). The effects of fatigue and sleepiness on nurse performance and patient safety. In R. G. Hughes (Ed.), Patient safety and quality: An evidencebased handbook for nurses (pp. 2-509-2-545). Rockville, MD: Agency for Healthcare Research and Quality.

Rogers, A. E., Hwang, W., Scott, L., Aiken, L., \& Dinges, D. (2004). The working hours of hospital staff nurses and patient safety. Health Affairs, 23(4), 202-212. doi:10.1377/hlthaff.23.4.202

Sabo, B. M. (2006). Compassion fatigue and nursing work: Can we accurately capture the consequences of caring work? International Journal of Nursing Practice, 12(3), 136-142. doi:10.1111/j.1440-172X.2006.00562.x

Seren, S., \& Baykal, U. (2007). Relationships between change and organizational culture in hospitals.
Journal of Nursing Scholarship, 39(2), 191-197. doi:10.1111/j.1547-5069.2007.00166.x

Sherman, R. O. (2019). 4 steps to repairing a toxic culture. Psychological safety, shared vision, staff ownership, and success celebration. American Nurse Today, 14(3), 5.

Sherman, R. O., \& Cohn, T. M. (2019). Promoting professional accountability and ownership. American Nurse Today, 14(2), 24-26.

Simkin, P., \& Ancheta, R. (2005). The labor progress handbook (2nd ed.). Oxford, UK: Blackwell Publishing.

Simpson, K. R. (2005). The context and clinical evidence for common nursing practices during labor. MCN: The American Journal of Maternal Child Nursing, 30(6), 356-363. doi:10.1097/00005721-200511000-00002

Simpson, K. R., \& Chez, B. F. (2001). Professional and legal issues. In K. R. Simpson \& P. A. Creehan (Eds.), AWHONN's perinatal nursing (2nd ed., pp. 21-52). Philadelphia, PA: Lippincott Williams \& Wilkins.

Sinclair, S., Beamer, K., Hack, T. F., McClement, S., Bouchal, S. R., Chochinov, H. M., \&Hagen,N. A. (2017). Sympathy, empathy, and compassion: A grounded theory study of palliative care patients' understandings, experiences, and preferences. Palliative Medicine, 31(5), 437-447. doi:10.1177/0269216316663499

Smith, J. (2019). Establishing norms of respect-Strategies for nurses and managers. American Nurse Today, 13(6), 44-46.

Stichler, J. F. (2006). Emotional intelligence. A critical leadership quality for the nurse executive. AWHONN Lifelines, 10(5), 422-425. doi:10.1111/j.1552-6356.2006.00083.x

Stichler, J. F. (2007a). Is your hospital hospitable? How physical environment influences patient safety. Nursing for Women's Health, 11(5), 506-511. doi:10.1111/j.1751-486X.2007.00226.x

Stichler, J. F. (2007b). Social intelligence: An essential trait of effective leaders. Nursing for Women's Health, 11(2), 189-193. doi:10.1111/j.1751-486X.2007.00144.x

Thompson, L., Krening, C., \& Parrett, D. (2018). Interdisciplinary team huddles for fetal heart rate tracing review. Nursing for Women's Health, 22(3), 240-249. doi:10.1016/j.nwh.2018.03.002

Thompson, R. (2019). What if you're the bully? Selfawareness and honest feedback can help you make a change. American Nurse Today, 14(1), 22-25.

Tolcher, M. C., Torbenson, V. E., Weaver, A. L., McGree, M. E., El-Nashar, S. A., Nesbitt, K. M., ...Famuyide, A. O. (2016). Impact of a labor and delivery safety bundle on a modified adverse outcomes index. American Journal of Obstetrics \& Gynecology, 214, 401.e1-401. e9. doi:10.1016/j.ajog.2016.01.155

Trinkoff, A., Geiger-Brown, J., Brady, B., Lipscomb, J. U., \& Muntaner, C. (2006). How long and how much are nurses now working? American Journal of Nursing, 106(4), 60-72. doi:10.1097/00000446-200604000-00030

Trossman, S. (2007). Issues up close: The science behind caring. American Nurse Today, 2(12), 40-41.

Tucker, S., Klotzbach, L., Olsen, G., Voss, J., Huus, B., \& Olsen, R. (2006). Lessons learned in translating research evidence on early intervention programs into clinical care. MCN: American Journal of Maternal Child Nursing, 31(5), 325-331. doi:10.1097/00005721-200609000-00012 
Welker-Hood, K. (2006). Does workplace stress lead to accident or error? American Journal of Nursing, 106(9), 104. doi:10.1097/00000446-200609000-00042

White, A. A., Pichert, J. W., Bledsoe, S. H., Irwin, C., \& Entman, S. S. (2005). Cause and effect analysis of closed claims in obstetrics and gynecology. Obstetrics \& Gynecology, 105(5, Pt. 1), 1031-1038. doi:10.1097.01.AOG.0000158864.09443.77

Whitehead, P. B., Herbertson, R. K., Hamric, A. B., Epstein, E. G., \& Fisher, J. M. (2015). Moral distress among healthcare professionals: Report of an institution-wide survey. Journal of Nursing Scholarship, 47(2), 117-125. doi:10.1111/jnu.12115

World Health Organization. (2018). WHO recommendations: Intrapartum care for a positive childbirth experience. Geneva, Switzerland: Author.

Zabari, M. L., \& Southern, N. L. (2018). Effects of shame and guilt on error reporting among obstetric clinicians. Journal of Obstetric, Gynecologic, and Neonatal Nursing, 47, 468-478. doi:10.1016/j.jogn.2018.03.002 